\title{
Final Year 5 Students' Exit Evaluation of the Asia Metropolitan University MBBS Program
}

\author{
Amaluddin Ahmad, Soe- Soe- Aye, Roy RilleraMarzo \\ Faculty of Medicine, Asia Metropolitan University, Johor, Malaysia \\ DOI: http://dx.doi.org/10.31344/ijhhs.v5i0.303
}

Introduction: An exit evaluation study by the final year graduating students was done Just prior to the completion of the third batch of the MBBS program at Asia Metropolitan University (AMU).

Objective: (1) To determine whether the MBBS program had enabled the students to (a)attain the eight Program Learning outcomes (PLO); (b)to achieve the eight major competency areas expected upon completion of the program; and (2) To look into the strengths and weaknesses of the program from the graduating final year students' perspectives.

Methods: A descriptive study was done among 18 medical students who were doing senior clerkship posting. A self-administered questionnaire including one open ended question was used for the study. Informed consent was obtained from the participants, assuring them on confidentiality. Data gathered were analysed using SPSS version 23.

Results: Most of the students (77\%) feel that the program had enabled them to attain each one of the eight Program Learning Outcomes(PLO); between 60-78\% achieved competencies in each one of the eight major areas expected at the end of course. The strengths included experienced lecturers, smooth implementation of the program, well designed curriculum while weakness was deficiency in clinical skills, training facilities, number of lecturers, case mix and adequacy of clinical exposure. The weaknesses include readiness to be a self-directed learner.

Conclusion: Majority of the students attained each of the eight (PLO) competencies. The strengths were identified and discussed. The study has paved the way for a more detailed indepth study with more samples among the future graduating batches.

Keywords: exit evaluation; medical education program; Asia Metropolitan University 